\title{
Stability-indicating high performance liquid chromatographic method for simultaneous assay of pibrentasvir and glecaprevir: Method development, validation and application to tablet dosage forms
}

\author{
Satya Venkata Sakuntala MAMIDI 1 * (D) , Lakshmana Rao ATMAKURI ${ }^{2}$ (D), \\ William Carey MAMIDIPALLI ${ }^{3}$ (D) \\ 1 Department of Pharmacy, Government Polytechnic for Women, Kakinada, and Research Scholar, JNTUniversity \\ Kakinada, India - 533003. \\ 2 Department of Pharmaceutical Analysis, V. V. Institute of Pharmaceutical Sciences, Gudlavalleru, Andhra Pradesh, \\ India - 521356. \\ 3 Department of Pharmacy, Government Polytechnic, Visakhapatnam, Andhra Pradesh, India - 530007. \\ * Corresponding Author. E-mail: m.sakuntala1@gmail.com (S.V.S.M.); Tel. +91-995-128 7148.
}

Received: 20 September 2018 / Revised: 29 November 2018/ Accepted: 07 December 2018

\begin{abstract}
Pibrentasvir and glecaprevir combination therapy acts by inhibiting RNA replication and viron assembly in hepatitis $C$ virus. The aim and objective of the present investigation is to develop and validate a stability indicating RP-HPLC method for simultaneous quantification of pibrentasvir and glecaprevir in bulk and tablets. Pibrentasvir and glecaprevir were separated and analyzed on Agilent Eclipse column $(4.6 \mathrm{~mm} \times 150 \mathrm{~mm}, 5 \mu \mathrm{m})$. The mobile phase consisted of $0.1 \%$ orthophosphoric acid and methanol $(30: 70, \mathrm{v} / \mathrm{v})$, that was isocratically delivered at a flow rate of 1.0 $\mathrm{mL} / \mathrm{min}$. Retention times were $1.857 \mathrm{~min}$ for glecaprevir and $2.681 \mathrm{~min}$ for pibrentasvir. Good regression coefficients were obtained in range of 50-250 $\mu \mathrm{g} / \mathrm{mL}$ for glecaprevir and $20-100 \mu \mathrm{g} / \mathrm{mL}$ for pibrentasvir. The results of validation parameters like selectivity, precision, accuracy and robustness are satisfactory. Tablet sample containing pibrentasvir and glecaprevir was subjected to degradation with $0.1 \mathrm{~N} \mathrm{HCl}, 0.1 \mathrm{~N} \mathrm{NaOH}, 30 \%$ hydrogen peroxide, thermal and photo conditions. The resulting degradants produced during the applied degradation conditions were well resolved from the peaks of pibrentasvir and glecaprevir. The utility of the proposed method was demonstrated by application to tablets containing pibrentasvir and glecaprevir combination. No interference from additives was observed. Therefore the method can be adapted in routine analysis of pibrentasvir and glecaprevir in quality control laboratories. The method can also be used for purity and degradation assessment of pibrentasvir and glecaprevir in tablets.
\end{abstract}

KEYWORDS: Antiviral drugs; pibrentasvir; glecaprevir; stability indicating; liquid chromatography.

\section{INTRODUCTION}

Hepatitis $C$ virus is an RNA virus which causes progressive damage to the liver. As a result, liver cirrhosis and hepatocellular carcinoma might occur. Approximately 64 to 103 million people are infected chronically with hepatitis C virus [1]. As per WHO (World Health Organization), 350000 to 500000 people die each year because of this virus associated liver diseases. Though this virus was found worldwide, majorly affected regions included North Africa, Central Asia and East Asia [2,3]. After better studying the properties of RNA, proteins and life cycle of hepatitis $C$ virus, effective antiviral treatments were developed. The duration of therapy with antiviral and probability of response to antiviral depends on the number and genotype of RNA in hepatitis $C$ virus [4].

The approval of pibrentasvir and glecaprevir combination was given by Food and Drug Administration in 2017 August [5]. This is used in healing the adult patients with chronic hepatitis $C$ virus genotypes 1 to 6 (with no/mild cirrhosis), with kidney disease, those patients on dialysis, patients infected with hepatitis C virus genotype 1 who were already treated either with an NS5A inhibitor or an NS3/4A protease inhibitor, but not both in the past [6-8]. The enzymes, nonstructural protease 3A (NS3A), 4A (NS4A) and 5A (NS5A) are

\footnotetext{
How to cite this article: Mamidi SVS, Atmakuri LR, Mamidipalli WC. Stability Indicating high performance liquid chromatographic method for simultaneous assay of pibrentasvir and glecaprevir: method development, validation and application to tablet dosage forms. J Res Pharm. 2019; 23(3): 465-475.
} 
associated with viron assembly and viral RNA replication. Pibrentasvir enacts as NS5A inhibitor while glecaprevir enacts as NS3/4A protease inhibitor. Thus, pibrentasvir and glecaprevir blocks arrest the growth of hepatitis $C$ virus through inhibition of its RNA replication and virion assembly [9]. The structure of pibrentasvir and glecaprevir are shown in Figure 1.
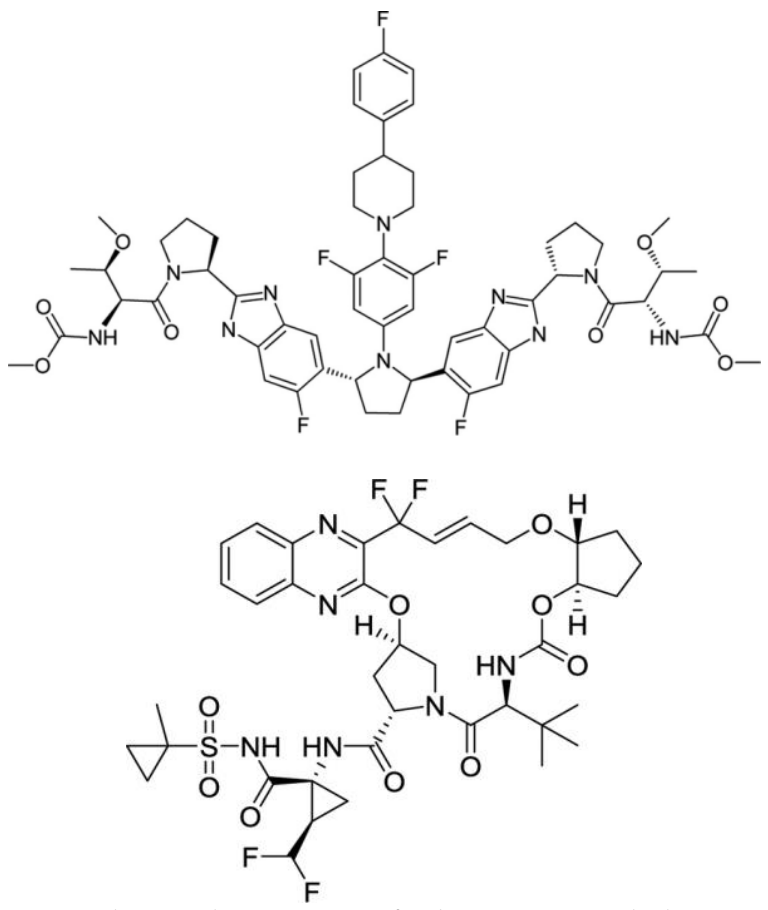

Figure 1. Chemical structures of pibrentasvir and glecaprevir.

In any pharmacopoeia, the combination of these two drugs is not included. Hence, there is a demand to establish an analytical method that can quantify pibrentasvir and glecaprevir simultaneously. Hitherto, only one HPLC [10] and one UPLC [11] methods are reported for the concurrent determination of pibrentasvir and glecaprevir. There is one method [12] established till date in simultaneous quantification of pibrentasvir and glecaprevir in bulk and tablet dosage forms using stability indicating RP-HPLC. The current study describes a novel, simple, precise and rapid stability indicating RP-HPLC method with UV detection for the simultaneous estimation of pibrentasvir and glecaprevir in bulk and in tablets with a run time of $10 \mathrm{~min}$ and less retention time.

\section{RESULTS}

\subsection{Optimization of HPLC conditions}

Three different columns were involved in performance investigations. They include Inertsil ODS C18 $(4.6 \mathrm{~mm} \times 150 \mathrm{~mm}, 5 \mu \mathrm{m})$, Symmetry C18 $(4.6 \mathrm{~mm} \times 150 \mathrm{~mm}, 5 \mu \mathrm{m})$ and Agilent Eclipse C18 column $(4.6 \mathrm{~mm}$ $\times 150 \mathrm{~mm}, 5 \mu \mathrm{m})$. The results revealed that the last column, Agilent Eclipse C18 column $(4.6 \mathrm{~mm} \times 150 \mathrm{~mm}$, $5 \mu \mathrm{m})$, was the most appropriate because it produced symmetrical peaks of glecaprevir and pibrentasvir with better resolution. The UV detector response of glecaprevir and pibrentasvir was recorded. The suitable detection wavelength was fixed at $244 \mathrm{~nm}$. At $244 \mathrm{~nm}$, both drugs showed good sensitivity with sensible response.

The effect of mobile phase composition, $\mathrm{pH}$ and flow rate on the resolution, retention time and peak symmetry of selected drugs was tested. For this purpose, different compositions (methanol: water, methanol: phosphate buffer, water: acetonitrile, phosphate buffer: acetonitrile and orthophosphoric acid: methanol) with different $\mathrm{pH}(3.0,3.5,4.0,4.5)$ and a flow rate $(0.8 \mathrm{~mL} / \mathrm{min}, 1.0 \mathrm{~mL} / \mathrm{min}, 1.2 \mathrm{~mL} / \mathrm{min})$ were tested. Finally, $0.1 \%$ orthophosphoric acid combined with methanol in the ratio $30: 70(\mathrm{v} / \mathrm{v})$, having flow rate $1.0 \mathrm{~mL} / \mathrm{min}$ and $\mathrm{pH} 3.5$ gave better resolution and sharp symmetric peaks with retention times of $1.857 \mathrm{~min} 2.681 \mathrm{~min}$ for glecaprevir and pibrentasvir, respectively Figure 2. 


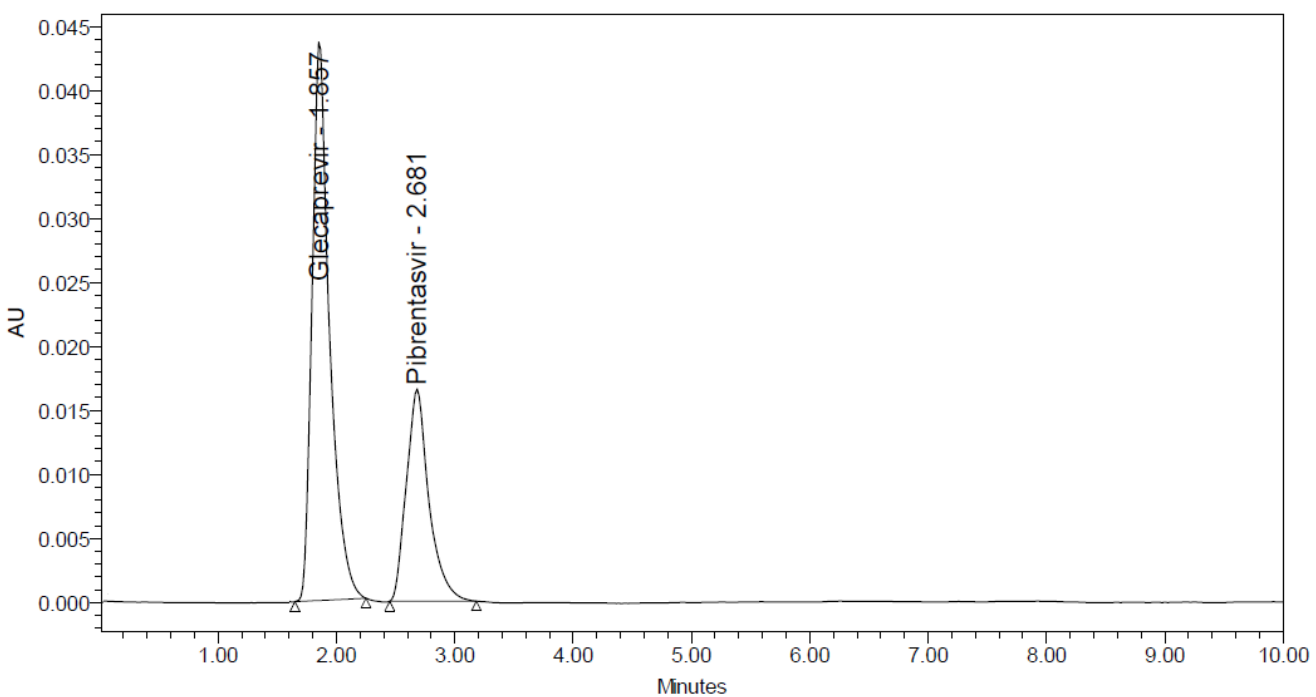

Figure 2. HPLC chromatogram of standard solution, containing glecaprevir and pibrentasvir with Agilent Eclipse column $(4.6 \times 150 \mathrm{~mm}, 5 \mu \mathrm{m})$ Mobile Phase: $0.1 \%$ orthophosphoric acid: methanol (30:70); flow rate $1 \mathrm{~mL} / \mathrm{min}$; detection wavelength $244 \mathrm{~nm}$; column temperature $25^{\circ} \mathrm{C}$.

\subsection{Method validation} [13-14].

International conference on harmonization guidelines was followed to validate the developed method

\subsubsection{System suitability}

System suitability was done with respect to injection repeatability (relative standard deviation of retention time and peak area response), tailing factor, theoretical plate number and resolution for glecaprevir and pibrentasvir peaks using a standard solution (glecaprevir $150 \mu \mathrm{g} / \mathrm{mL}$ and pibrentasvir $60 \mu \mathrm{g} / \mathrm{mL}$ ). Data summarised in Table1 shows satisfactory results for System suitability. The relative standard deviation of retention time and peak area response of 5 consecutive injections was observed as $<1.0 \%$ for both drugs, indicating excellent injection repeatability. The tailing factor was found to be 1.463 (glecaprevir) and 1.287 (pibrentasvir), reflecting good peak symmetry. The theoretical plate number was found to be $>4000$ for both drugs which demonstrate satisfactory column efficiency. Finally, the resolution values of 3.183 show the good separation of glecaprevir and pibrentasvir peaks. The results assure the adequacy of the system for the simultaneous analysis of glecaprevir and pibrentasvir.

Table 1. System suitability data for the analysis of glecaprevir and pibrentasvir.

\begin{tabular}{lcccc}
\hline & \multicolumn{2}{c}{ Glecaprevir } & \multicolumn{2}{c}{ Pibrentasvir } \\
\cline { 2 - 5 } Parameter & Value* & RSD & Value* & RSD \\
\hline Retention & 1.857 & 0.164 & 2.681 & 0.114 \\
time(min) & & & & \\
Tailing factor & 1.463 & 0.395 & 1.287 & 0.449 \\
Plate number & 4738 & 0.547 & 6354 & 0.135 \\
Resolution & - & - & 3.183 & 0.181 \\
\hline${ }^{*}$ Average of five determinations. & & &
\end{tabular}

\subsubsection{Selectivity}

Selectivity of method was assessed by checking that no interference peaks were found at the retention times of glecaprevir and pibrentasvir with mobile phase blank and tablet sample solutions. For this, chromatograms of solutions of standard (glecaprevir $150 \mu \mathrm{g} / \mathrm{mL}$ and pibrentasvir $60 \mu \mathrm{g} / \mathrm{mL}$ ), tablet sample (glecaprevir $150 \mu \mathrm{g} / \mathrm{mL}$ and pibrentasvir $60 \mu \mathrm{g} / \mathrm{mL}$ ) and mobile phase blanks are compared. The chromatograms of standard and tablet sample showed peaks for glecaprevir and pibrentasvir without any interfering peak Figure 3. In mobile phase blank chromatogram Figure 3, no peak was observed at the retention times of glecaprevir and pibrentasvir. Thus the method was proved selective. 

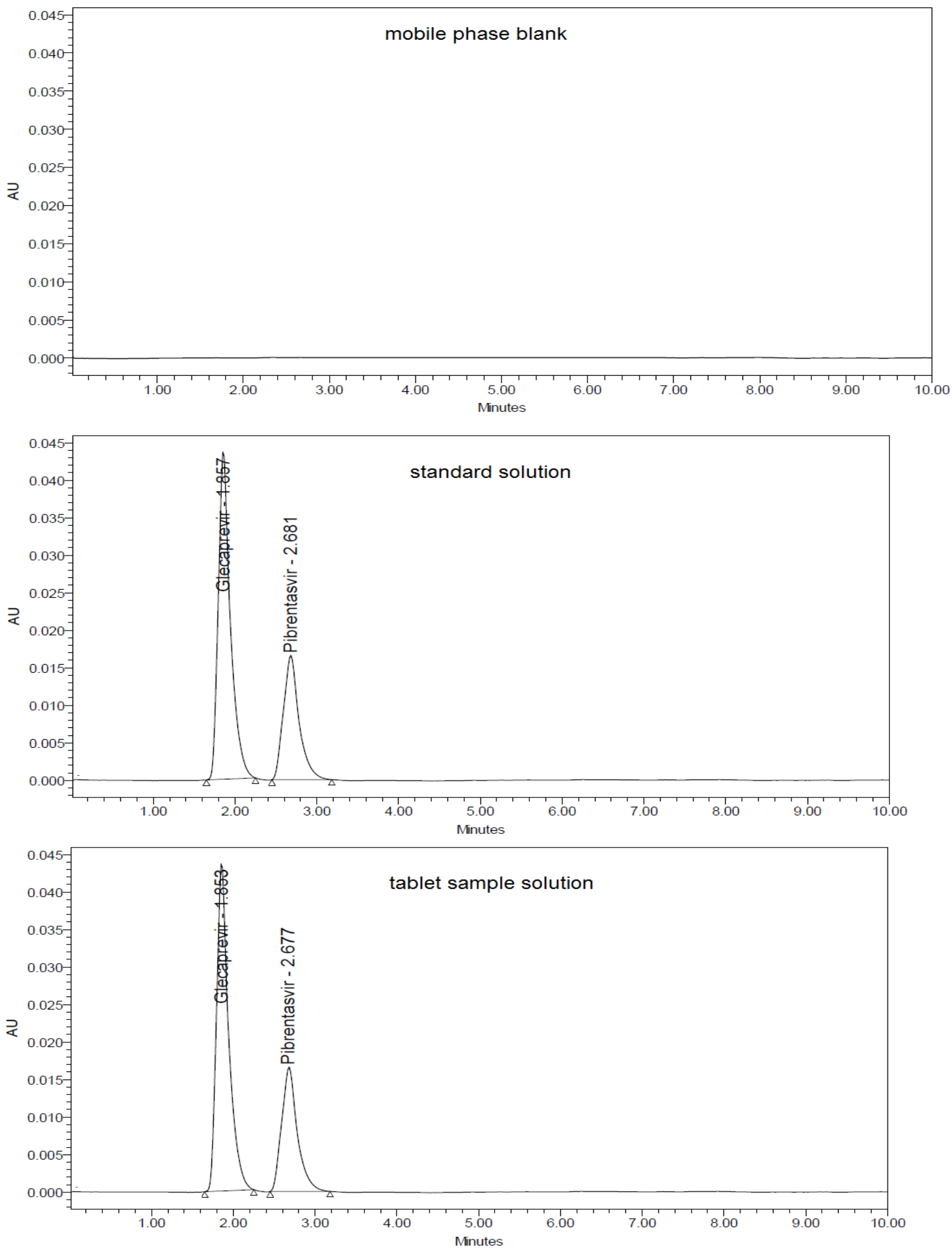

Figure 3. Selectivity study chromatograms. 


\subsubsection{Linearity}

Calibration curves were linear over a glecaprevir concentration range of 50 to $250 \mu \mathrm{g} / \mathrm{mL}$ and pibrentasvir concentration range of 20 to $100 \mu \mathrm{g} / \mathrm{mL}$. Linear regression of glecaprevir and pibrentasvir curves resulted in a linear fit of $y=3008 x-6630\left(R^{2}=0.9996\right)$ and $y=3586 x-608.5\left(R^{2}=0.9998\right)$, respectively. The results demonstrated good linearity of glecaprevir and pibrentasvir calibration curves.

\subsubsection{Limit of detection and limit of quantitation}

A signal to noise ratio of 3:1 and 10:1 is accepted to calculate the limit of detection (LOD) and limit of quantitation (LOQ), respectively. The limit of detection was calculated as $0.60 \mu \mathrm{g} / \mathrm{mL}$ for both drugs. The limit of quantitation was calculated as $1.95 \mu \mathrm{g} / \mathrm{mL}$ and $2.01 \mu \mathrm{g} / \mathrm{mL}$ for glecaprevir and pibrentasvir, respectively. Therefore, the method is sensible for glecaprevir and pibrentasvir analysis in tablet formulations. Chromatograms at LOD and LOQ level are shown in Figure 4.
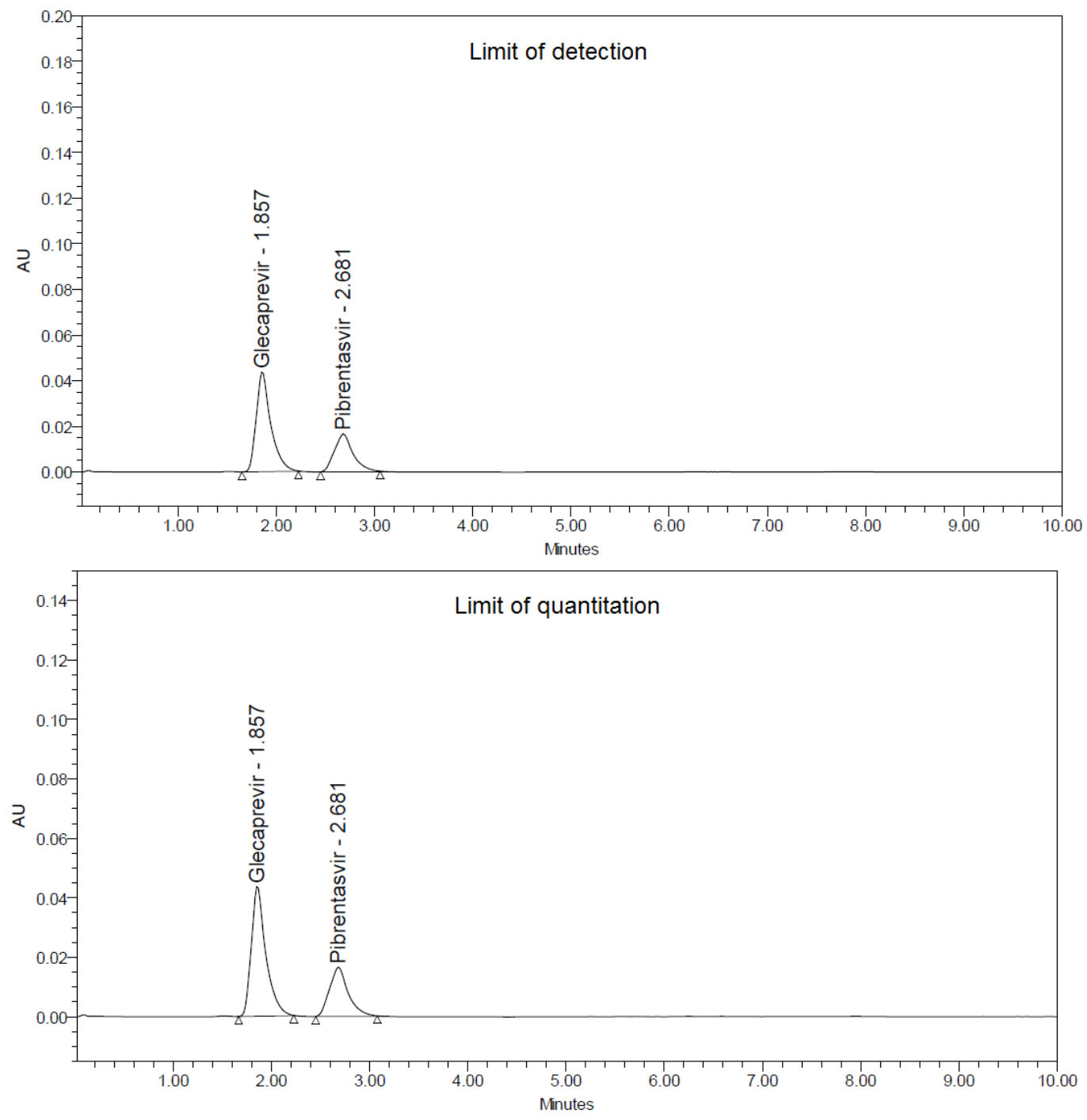

Figure 4. Chromatograms of glecaprevir and pibrentasvir at LOD and LOQ level.

\subsubsection{Precision}

System precision was tested using a standard solution with concentration $150 \mu \mathrm{g} / \mathrm{mL}$ and $60 \mu \mathrm{g} / \mathrm{mL}$ of glecaprevir and pibrentasvir, respectively. Data summarized in Table 2 revealed satisfactory values for system precision. The Relative standard deviation of peak area response was $0.203 \%$ for glecaprevir and $0.677 \%$ for pibrentasvir.

Method precision was assessed using tablet sample solution having concentration $150 \mu \mathrm{g} / \mathrm{mL}$ of glecaprevir and $60 \mu \mathrm{g} / \mathrm{mL}$ of pibrentasvir. Relative standard deviation of the percent assay of glecaprevir and 
pibrentasvir were calculated. The data shown in Table 2 revealed satisfactory values for method precision. The relative standard deviation of the percent assay was $0.416 \%$ for glecaprevir and $0.473 \%$ for pibrentasvir.

Evaluation of intermediate precision/ruggedness of the method was performed using glecaprevir and pibrentasvir standard solution with concentration $150 \mu \mathrm{g} / \mathrm{mL}$ and $60 \mu \mathrm{g} / \mathrm{mL}$, respectively on two different days. Peak area values for glecaprevir and pibrentasvir along with mean and percent relative standard deviation values are summarized in Table 2. Relative standard deviation of peak area response was less than $1 \%$ for glecaprevir and pibrentasvir indicating the acceptable values for intermediate precision/ruggedness of the method.

Table 2. Data of system, method, intermediate precision experiments.

\begin{tabular}{ccccc}
\hline \multirow{2}{*}{$\begin{array}{c}\text { Sample } \\
\text { injection }\end{array}$} & \multicolumn{2}{c}{ System precision } & \multicolumn{2}{c}{ Method precision } \\
\cline { 2 - 5 } & \multicolumn{2}{c}{ Peak area } & Glecaprevir & Pibrentasvir \\
\cline { 2 - 5 } & Glecaprevir & Pibrentasvir & 99.00 & 100.52 \\
\hline 1 & 448662 & 218753 & 99.79 & 99.67 \\
3 & 446873 & 214829 & 99.32 & 100.10 \\
4 & 446352 & 216426 & 99.53 & 99.90 \\
5 & 447562 & 218452 & 100.00 & 100.18 \\
6 & 447529 & 216468 & 98.99 & 99.15 \\
Mean & 446244 & 217567 & 99.44 & 99.92 \\
$\%$ RSD & 447203.7 & 217082.5 & 0.416 & 0.473 \\
& 0.203 & 0.677 & Pibrentasvir peak area \\
& Intermediate precision/Ruggedness & Day 2 \\
1 & Glecaprevir peak area & Day 1 & 217584 \\
2 & Day 1 & Day 2 & 218573 & 217685 \\
3 & 448776 & 446582 & 218562 & 216452 \\
4 & 445735 & 445154 & 214652 & 218574 \\
5 & 447673 & 443565 & 215354 & 216854 \\
6 & 448673 & 446352 & 216454 & 214578 \\
Mean & 445876 & 447545 & 216457 & 216955 \\
$\%$ RSD & 448676 & 446585 & 216675 & 0.634 \\
\hline
\end{tabular}

\subsubsection{Accuracy}

Accuracy was demonstrated in terms of recovery of known concentrations of glecaprevir and pibrentasvir spiked to preanalyzed tablet sample solution at three levels $(50 \%, 100 \%$ and $150 \%$ of labeled claim). Calculated recoveries of glecaprevir and pibrentasvir at each level are shown in Table 3. The results suggest the acceptable accuracy of the developed method and non-interference of tablet excipients in the assay.

Table 3. Data of accuracy experiments.

\begin{tabular}{cccccc}
\hline Drug & $\begin{array}{c}\text { Level tested } \\
\mathbf{\%}\end{array}$ & $\begin{array}{c}\text { Labeled } \\
\text { amount(mg) }\end{array}$ & $\begin{array}{c}\text { Amount } \\
\text { added }(\mathbf{m g})\end{array}$ & $\begin{array}{c}\text { Amount } \\
\text { Found(mg) }\end{array}$ & Recovery(\%) \\
\hline Glecaprevir & 50 & 100 & 50 & 151.05 & 100.70 \\
& 100 & 100 & 100 & 200.08 & 100.04 \\
& 150 & 100 & 150 & 251.12 & 100.45 \\
\hline Pibrentasvir & 50 & 40 & 20 & 60.27 & 100.45 \\
& 100 & 40 & 40 & 80.40 & 100.50 \\
& 150 & 40 & 60 & 100.23 & 100.23 \\
\hline
\end{tabular}


Table 4. Recovery, degradation and peak purity data of glecaprevir and pibrentasvir in applied stress conditions.

\begin{tabular}{|c|c|c|c|c|c|c|}
\hline Drug & Condition & Peak area & $\begin{array}{c}\% \\
\text { Recovery }\end{array}$ & $\begin{array}{c}\% \\
\text { degraded }\end{array}$ & $\begin{array}{l}\text { Purity } \\
\text { angle }\end{array}$ & $\begin{array}{c}\text { Purity } \\
\text { Threshold }\end{array}$ \\
\hline \multirow{7}{*}{ Glecaprevir } & Undegraded & 447408 & 100 & - & - & - \\
\hline & Acid & 436572 & 97.57 & 2.43 & 0.589 & 1.619 \\
\hline & Base & 428673 & 95.81 & 4.19 & 0.865 & 3.529 \\
\hline & & & & & & \\
\hline & Peroxide & 439657 & 98.27 & 1.73 & 0.426 & 0.964 \\
\hline & Thermal & 430876 & 96.30 & 3.70 & 0.379 & 1.146 \\
\hline & Photo & 421862 & 94.29 & 5.71 & 0.786 & 2.744 \\
\hline \multirow{7}{*}{ Pibrentasvir } & Undegraded & 217707 & 100 & - & - & - \\
\hline & Acid & 207853 & 95.47 & 4.53 & 0.167 & 0.354 \\
\hline & Base & 196762 & 90.38 & 9.62 & 0.264 & 0.589 \\
\hline & & & & & & \\
\hline & Peroxide & 206752 & 94.97 & 5.03 & 0.638 & 1.532 \\
\hline & Thermal & 199672 & 91.72 & 8.28 & 0.397 & 0.733 \\
\hline & Photo & 195534 & 89.92 & 10.18 & 0.369 & 0.846 \\
\hline
\end{tabular}

\subsubsection{Specificity}

The peak area of glecaprevir and pibrentasvir in the stress degraded samples was less than the peak area of glecaprevir and pibrentasvir in standard solution indicating that the selected drugs

undergoes partial degradation in all applied conditions. Peak area and \% recoveries of glecaprevir and pibrentasvir remaining after applying degradation processes were calculated and summarized in Table 4 . As per the results shown in Table 4, the glecaprevir and pibrentasvir are more susceptible towards photolysis condition. The chromatograms of degradation studies are shown in Figure 5. Chromatogram of tablet treated with $0.1 \mathrm{~N} \mathrm{HCl}$ shows one peak for unknown degradation product with retention time $5.256 \mathrm{~min}$. Chromatograms of tablet treated with $0.1 \mathrm{~N} \mathrm{NaOH}$ and $30 \%$ hydrogen peroxide showing peaks for three unknown degradation products (retention time of degradants - $1.182 \mathrm{~min}, 4.406 \mathrm{~min}$ and $6.918 \mathrm{~min}$ in alkaline condition; $3.390 \mathrm{~min}, 5.256 \mathrm{~min}$ and $6.585 \mathrm{~min}$ in oxidative condition). Chromatogram of tablet undergone thermal and photo degradation shows peaks for 2 unknown degradation products (retention time of degradants - $3.685 \mathrm{~min}$ and $4.923 \mathrm{~min}$ in thermal degradation; $5.256 \mathrm{~min}$ and $7.260 \mathrm{~min}$ in photo degradation). The proposed method was able to detect and specifically quantitate glecaprevir and pibrentasvir peak in the presence of degradants. The results indicating that the proposed method can be used as a stability indicating method. In the applied forced degradation conditions, the purity of glecaprevir and pibrentasvir peaks were confirmed by the higher values of peak threshold value than peak purity angle acquired through the peak purity tool Table 4. 


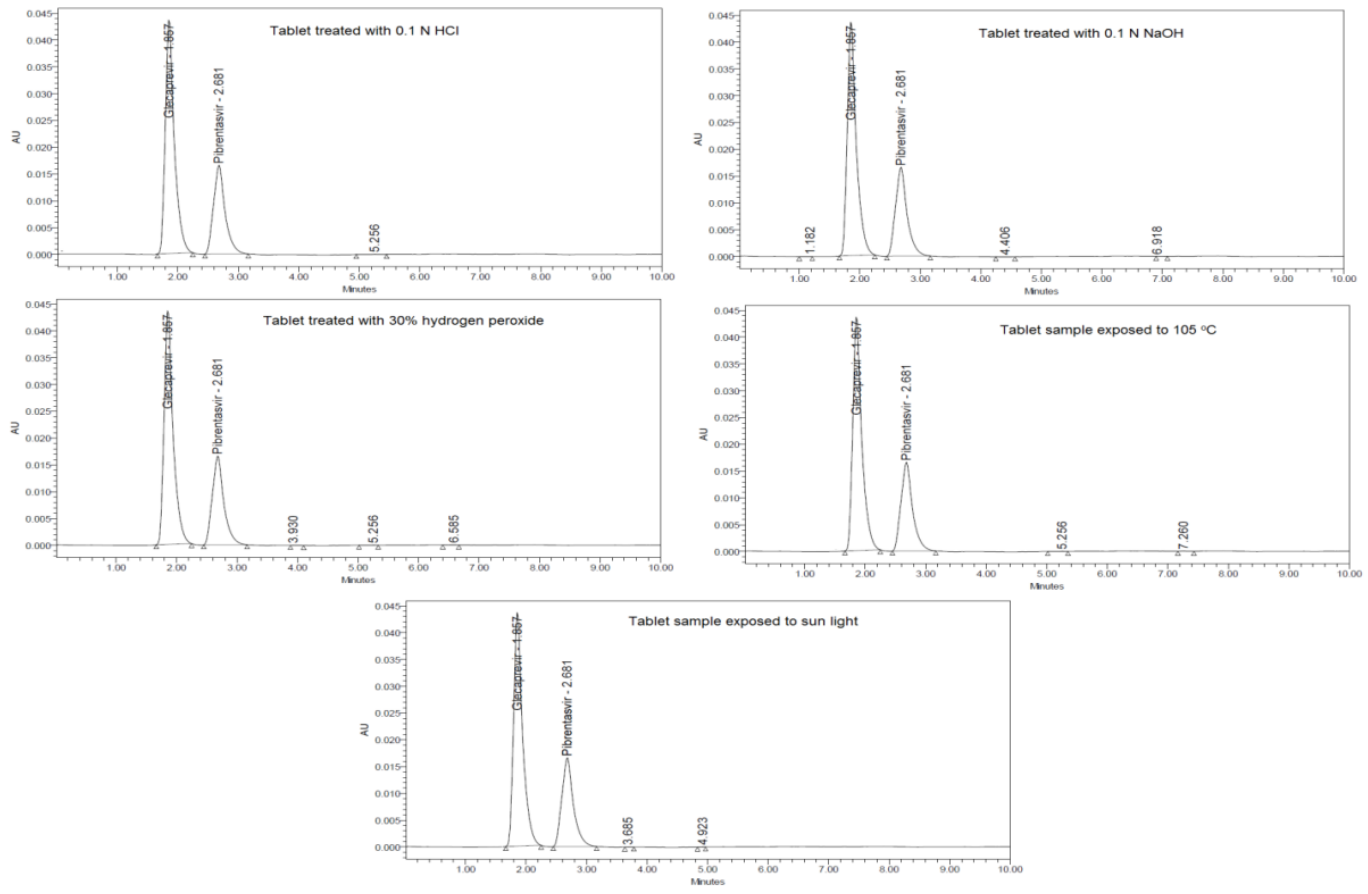

Figure 5. Chromatograms of degradation studies.

\subsubsection{Robustness}

During method robustness testing, deliberate change in the flow rate, mobile phase composition, $\mathrm{pH}$ of the mobile phase, detection wavelength and temperature variation was made to evaluate the impact on the system suitability parameters of the developed method. Results are presented in Table 5. No significant changes were observed in studied system suitability parameters when deliberate variations are made in the chromatographic conditions which mean that the proposed method is robust.

Table 5. Data of method robustness tests.

\begin{tabular}{cccccccc}
\hline $\begin{array}{c}\text { Chromatographic } \\
\text { Condition }\end{array}$ & Value & \multicolumn{2}{c}{ Glecaperavir } & Resolution & \multicolumn{2}{c}{ Pibrentasvir } \\
\cline { 3 - 4 } & & $\begin{array}{c}\text { Tailing } \\
\text { factor }\end{array}$ & $\begin{array}{c}\text { Plate } \\
\text { count }\end{array}$ & & & $\begin{array}{c}\text { Tailing } \\
\text { factor }\end{array}$ & $\begin{array}{c}\text { Plate } \\
\text { count }\end{array}$ \\
\hline Flow & 0.9 & 1.463 & 4626.92 & 3.31 & 1.295 & 6132.29 \\
rate $(\mathrm{mL} / \mathrm{min})$ & 1.0 & 1.465 & 4725.92 & 3.18 & 1.293 & 6256.39 \\
& 1.1 & 1.466 & 4865.39 & 3.02 & 1.291 & 6352.29 \\
\hline Mobile phase & $25: 75$ & 1.467 & 4762.23 & 3.37 & 1.299 & 6214.27 \\
cpmposition ratio & $30: 70$ & 1.462 & 4725.92 & 3.18 & 1.292 & 6256.39 \\
(v/v) & $35: 65$ & 1.464 & 4767.76 & 2.96 & 1.295 & 6232.23 \\
\hline $\mathrm{p}^{\mathrm{H}}$ of mobile & 3.3 & 1.469 & 4568.25 & 3.35 & 1.298 & 5895.25 \\
phase & 3.5 & 1.462 & 4725.92 & 3.18 & 1.294 & 6256.39 \\
& 3.7 & 1.460 & 4635.27 & 2.95 & 1.292 & 6145.95 \\
\hline Detection wave & 240 & 1.468 & 4635.25 & 3.18 & 1.293 & 6065.32 \\
length $(\mathrm{nm})$ & 244 & 1.460 & 4725.92 & 3.18 & 1.296 & 6256.39 \\
& 248 & 1.461 & 4762.35 & 3.18 & 1.297 & 6264.85 \\
\hline Column & 23 & 1.466 & 4578.35 & 3.33 & 1.292 & 6145.35 \\
temperature $(\mathrm{oC})$ & 25 & 1.464 & 4725.92 & 3.18 & 1.295 & 6256.39 \\
& 27 & 1.465 & 4658.26 & 3.04 & 1.298 & 6758.15 \\
\hline
\end{tabular}

\subsection{Application of the method}

The developed and validated assay method was applied to tablets containing glecaprevir and pibrentasvir. The mean concentration and recoveries for glecaprevir and pibrentasvir were found to be in good agreement with the nominal values Table 6 . The method was also precise with relative standard deviation value $<0.5 \%$. 
Table 6. Assay data of glecaprevir and pibrentasvir by the proposed method.

\begin{tabular}{cccccc}
\hline Glecaprevir & \multicolumn{5}{c}{ Pibrentasvir } \\
\hline $\begin{array}{c}\text { Labeled } \\
\text { claim(mg) }\end{array}$ & $\begin{array}{c}\text { Amount } \\
\text { found(mg) }\end{array}$ & $\begin{array}{c}\text { Recovery } \\
\mathbf{( \% )}\end{array}$ & $\begin{array}{c}\text { Labeled } \\
\text { claim(mg) }\end{array}$ & $\begin{array}{c}\text { Amount } \\
\text { found(mg) }\end{array}$ & $\begin{array}{c}\text { Recovery } \\
\mathbf{( \% )}\end{array}$ \\
\hline 100 & 100.19 & 100.19 & 40 & 39.95 & 99.88 \\
100 & 99.95 & 99.95 & 40 & 40.18 & 100.45 \\
100 & 99.93 & 99.93 & 40 & 40.05 & 100.13 \\
\hline Mean & 100.02 & 100.02 & Mean & 40.06 & 100.15 \\
\%RSD & 0.145 & 0.145 & \%RSD & 0.288 & 0.288 \\
\hline
\end{tabular}

\section{CONCLUSION}

A stability indicating RP-HPLC method was developed for the determination of glecaprevir and pibrentasvir simultaneously. The developed method yielded satisfactory validation results concerning linearity, sensitivity, precision, accuracy and ruggedness. The present method for the assay of glecaprevir and pibrentasvir in tablets is specific and selective since the tablet excipients and degradants produced did not interfere with the peaks of selected drugs. The method can be adapted in quality control laboratories for the quantification of glecaprevir and pibrentasvir.

\section{MATERIALS AND METHODS}

\subsection{Instrumentation}

- HPLC system: Waters 2695 HPLC system provided with high speed auto sampler, column, oven, degasser and 2996 photodiode array detector.

- Software for data processing: Waters Empower 2 software.

- pH meter: Adwa AD 1020, Adwa Hungary Kft, Hungary

- Weighing balance: Afcoset ER-200A, Mumbai, India.

\subsection{Materials}

\subsubsection{Reference standards}

- Pibrentasvir: Pharmatrain, Hyderabad, India (procured as gift sample)

- Glecaprevir: Pharmatrain, Hyderabad, India (procured as gift sample)

\subsubsection{Tablets}

- $\quad$ Maviret ${ }^{\circledR}$ tablets: manufactured by AbbVie Limited, Berkshire, UK. Labeled to contain $100 \mathrm{mg}$ and $40 \mathrm{mg}$ of glecaprevir and pibrentasvir, respectively.

\subsubsection{Chemicals}

- Analytical reagent grade orthophosphoric acid (Merck chemical division, Mumbai, India)

- Analytical reagent grade hydrochloric acid (SD Fine Chemicals Ltd, Chennai, India)

- Analytical reagent grade sodium hydroxide (SD Fine Chemicals Ltd, Chennai, India)

- Analytical reagent grade hydrogen peroxide (SD Fine Chemicals Ltd, Chennai, India)

- HPLC grade methanol (Merck chemical division, Mumbai, India)

- HPLC grade water (Milli-Q water purification system, Bangalore, India)

\subsection{Chromatographic conditions}

- Column

- Temperature

- Mobile phase

- $\mathrm{pH}$ of mobile phase

- Detection wavelength
: Agilent Eclipse column C18 (4.6 mm × $150 \mathrm{~mm}, 5 \mu \mathrm{m})$

: $25{ }^{\circ} \mathrm{C}$

: $30 \% 0.1 \%$ orthophosphoric acid : $70 \%$ methanol (degassed and filtered before use)

: 3.5

: $244 \mathrm{~nm}$ 
- Flow rate

- Injection volume

- Run time

$$
\begin{aligned}
& 1 \mathrm{~mL} / \mathrm{min} \\
& 20 \mu \mathrm{L} \\
& 10 \mathrm{~min}
\end{aligned}
$$

\subsection{Standard solutions}

Stock standard solution containing $1000 \mu \mathrm{g} / \mathrm{mL}$ and $400 \mu \mathrm{g} / \mathrm{mL}$ concentration of glecaprevir and pibrentasvir, respectively was prepared by dissolving appropriate quantities of drugs in the diluent $(0.1 \%$ orthophosphoric acid: methanol in the ratio $30: 70 \mathrm{v} / \mathrm{v}$ ). Serial dilutions of the stock standard solution were made with diluent to get working standard solutions at concentrations of 50, 100, 150, $200 \mathrm{and} 250 \mu \mathrm{g} / \mathrm{mL}$ of glecaprevir, and 20,40, 60, 80 and $100 \mu \mathrm{g} / \mathrm{mL}$ of pibrentasvir.

\subsection{Calibration curve}

The standard solution (concentration: glecaprevir 50-250 $\mu \mathrm{g} / \mathrm{mL}$ and pibrentasvir 20-100 $\mu \mathrm{g} / \mathrm{mL}$ ) was filtered through 0.44 micron syringe filters. The solutions were injected into the system with constant injection volume $(20 \mu \mathrm{L})$. Calibration curve was plotted between peak area and respective concentration. The regression coefficient and slope were determined from the calibration curve.

\subsection{Assay of selected drug combination in tablet}

Five Maviret ${ }^{\circledR}$ tablets were crushed into fine powder. Powder equivalent to $100 \mathrm{mg}$ glecaprevir and 40 $\mathrm{mg}$ pibrentasvir was accurately weighed and dissolved in $70 \mathrm{~mL}$ of diluent in a $100 \mathrm{~mL}$ volumetric flask. The content of the flask was sonicated for ten min to dissolve the drugs completely followed by dilution up to the mark with the same solvent. Pipette $1.0 \mathrm{~mL}$ of the prepared solutions into a $10 \mathrm{~mL}$ volumetric flask and dilute up to the mark with diluent (concentration: $100 \mu \mathrm{g} / \mathrm{mL}$ glecaprevir and $40 \mu \mathrm{g} / \mathrm{mL}$ pibrentasvir). $20 \mu \mathrm{L}$ of the prepared tablet solution was injected $(n=3)$ into the chromatographic system. Using the proposed method, measure the peak areas for glecaprevir and pibrentasvir and calculate their content in the tablet.

\subsection{Forced degradation study}

Forced degradation study was executed to analyze the method's stability indicating property, method's specificity and inherent stability characteristics of glecaprevir and pibrentasvir. Degradation was done by exposing the tablet solution $(1500 \mu \mathrm{g} / \mathrm{mL}$ glecaprevir and $600 \mu \mathrm{g} / \mathrm{mL}$ pibrentasvir) to 5 stress conditions (acid, alkali, hydrogen peroxide, thermal and photo) [13]. Stressed samples were analyzed and the presence of related peaks and peak purity for glecaprevir and pibrentasvir was checked.

\subsubsection{Hydrolytic degradation using $0.1 \mathrm{~N} \mathrm{HCl}$}

$1.5 \mathrm{~mL}$ of tablet solution was transferred into a $10 \mathrm{~mL}$ volumetric flask and $3 \mathrm{~mL}$ of $0.1 \mathrm{~N} \mathrm{HCl}$ was added. The flask was refluxed at $60^{\circ} \mathrm{C}$ for 24 hours, neutralized with $0.1 \mathrm{~N} \mathrm{NaOH}$ and make up to $10 \mathrm{~mL}$ with diluent. The solution was filtered with 0.44 micron syringe filters and injected into the system.

\subsubsection{Hydrolytic degradation using $0.1 \mathrm{~N} \mathrm{NaOH}$}

Tablet solution $(1.5 \mathrm{~mL})$ was transferred into a $10 \mathrm{~mL}$ volumetric flask followed by the addition of $3 \mathrm{~mL}$ of $0.1 \mathrm{~N} \mathrm{NaOH}$. The flask was refluxed at $60^{\circ} \mathrm{C}$ for 24 hours, neutralized with $0.1 \mathrm{~N} \mathrm{HCl}$ and diluted to $10 \mathrm{~mL}$ with diluent. The solution was filtered using 0.44 micron syringe filters and injected into the system.

\subsubsection{Oxidative degradation using $30 \%$ hydrogen peroxide}

$1.5 \mathrm{~mL}$ of tablet solution and $1 \mathrm{~mL}$ of $30 \%$ hydrogen peroxide was added to a $10 \mathrm{~mL}$ volumetric flask. The volume was made up to the mark with diluent. The contents of the flask were kept at room temperature for $15 \mathrm{~min}$. The solution was filtered using 0.44 micron syringe filters and injected into the system.

\subsubsection{Thermal induced degradation}

Tablet powder was taken in a petridish and kept in hot air oven set at $110^{\circ} \mathrm{C}$ for $3 \mathrm{~h}$. Tablet powder equivalent to $100 \mathrm{mg}$ glecaprevir and $40 \mathrm{mg}$ pibrentasvir was transferred into a $100 \mathrm{~mL}$ volumetric flask. Add about $70 \mathrm{~mL}$ of diluent and sonicated for ten min and volume was made up to the mark with diluent. $1.5 \mathrm{~mL}$ of the above prepared solution was diluted to $10 \mathrm{~mL}$ with the diluent. The resulting solution was filtered using 0.44 micron syringe filters and injected into the system. 


\subsubsection{Photodegradation using sunlight}

$1.5 \mathrm{~mL}$ of tablet solution was transferred into a $10 \mathrm{~mL}$ volumetric flask and exposed to sunlight for 24 $\mathrm{h}$. The solution was filtered using 0.45 micron syringe filters and injected into the system.

Acknowledgements: The authors are thankful to Pharmatrain, Hyderabad, India for providing gift samples of standards, glecaprevir and pibrentasvir.

Author contributions: Concept - S.V.S.M., W.C.M.; Design -S.V.S.M., L.R.A., W.C.M.; Supervision - L.R.A., W.C.M.; Resource - S.V.S.M. ,L.R.A., W.C.M.; Materials - S.V.S.M., L.R.A., W.C.M.; Data Collection and/or Processing S.V.S.M.; Analysis and/or Interpretation - S.V.S.M., L.R.A., W.C.M.; Literature Search - S.V.S.M., L.R.A., W.C.M.; Writing - S.V.S.M., L.R.A.,W.C.M.; Critical Reviews - S.V.S.M., L.R.A.,W.C.M.

Conflict of interest statement: The authors declare that no conflict of interest.

\section{REFERENCES}

[1] Manns MP, Buti M, Gane E, Pawlotsky JM, Razavi H, Terrault N, Younossi Z. Hepatitis C virus infection. Nat Rev Dis Primers. 2017; 3: 17006. [CrossRef]

[2] Bastos JCS, Padilla MA, Caserta LC, Miotto N, Vigani AG, Arns CW. Hepatitis C virus: Promising discoveries and new treatments. World J Gastroenterol. 2016; 22(28): 6393-6401. [CrossRef]

[3] World Health Organization. Hepatitis C, WHO fact sheet No. 164, updated July 2015. Available from: [CrossRef]

[4] Li HC, Lo SY. Hepatitis C virus: Virology, diagnosis and treatment. World J Hepatol. 2015; 7(10): 1377-1389. [CrossRef]

[5] Lamb YN. Glecaprevir/Pibrentasvir: First global approval. Drugs. 2017; 77(16): 1797-1804. [CrossRef]

[6] Forns X, Lee SS, Valdes J, Lens S, Ghalib R, Aguilar H, Felizarta F, Hassanein T, Hinrichsen H, Rincon D, Morillas R, Zeuzem S, Horsmans Y, Nelson DR, Yu Y, Krishnan P, Lin CW, Kort JJ, Mensa FJ. Glecaprevir plus pibrentasvir for chronic hepatitis $C$ virus genotype $1,2,4,5$, or 6 infection in adults with compensated cirrhosis (EXPEDITION-1): a single-arm, open-label, multicentre phase 3 trial. Lancet Infect Dis. 2017; 17(10): 1062-1068. [CrossRef]

[7] Levien TL, Baker DE. Formulary drug reviews: Glecaprevir/Pibrentasvir. Hosp Pharm. 2018; 53(2): 75-84. [CrossRef]

[8] Aghemo A, Colombo M. Glecaprevir/Pibrentasvir: The final piece in the Hepatitis C virus treatment puzzle?. Gastroenterology. 2018; 154(4): 1195-1196. [CrossRef]

[9] Ara AK, Paul JP. Novel emerging treatments for hepatitis C infection: A fast-moving pipeline. Therap Adv Gastroenterol. 2017; 10(2): 277-282. [CrossRef]

[10] Hemalatha K, Kistayya C, Nizamuddhin ND, Dastiagiriamma D. Simultaneous estimation of new analytical method development and validation of glecaprevir and pibrentasvir by high performance liquid chromatography. Innovat Int J Med Pharma Sci. 2018; 3(s1): 5-8.

[11] Sridevi M, Rao TS, Naidu CG. Development and validation for the simultaneous estimation of glecaprevir and pibrentasavir in drug product by UPLC. Eur J Biomed Pharm Sci. 2018; 5 (4): 473-480.

[12] China Babu M, Madhusudhana Chetty C, Mastanamma SK. A new force indicating RP-HPLC method development and validation for the simultaneous estimation of pibrentasvir and glecaprevir in bulk and its tablet dosage form. Pharm Methods. 2018; 9 (2): 79-86. [CrossRef]

[13] International conference on the harmonization. ICH Harmonized Tripartite Guideline. Validation of Analytical Procedures: Text and Methodology Q2 (R1), November 2005.

[14] International conference on the harmonization. ICH Harmonized Tripartite Guideline. Stability Testing of New Drug Substances and Products Q1A (R2), November 2003. 